\title{
DIAGNÓSTICO DE DEMÊNCIA, DEPRESSÃO E PSICOSE EM IDOSOS POR AVALIAÇÃO COGNITIVA BREVE
}

\author{
Bruno Netto dos Reys, Ana Beatriz Bezerra, Ana lucia de Sousa Vilela, Alexandre lins Keusen, Valeska Marinho, Estevão de Paula, Jerson laks*
}

Trabalho realizado no Centro Psiquiátrico Rio de Janeiro / Centro para Doença de Alzheimer do Instituto de Psiquiatria da UFRJ

*Correspondência:

Av. N. S. Copacabana, $749 / 802$

Copacabana - 22050-000

Rio de Janeiro - RJ

¡laks@centroin.com.br

\section{RESUMO}

Oвjetivo. Comparar o desempenho cognitivo de idosos com psicose, depressão e demência em um ambulatório de saúde mental.

Métodos. Aplicou-se o miniexame do estado mental (MEEM) e o Cambridge Cognitive Examination (CAMCOG) em 86 pacientes com mais de 60 anos de idade encaminhados para avaliação por queixas de memória. Os pacientes foram diagnosticados seguindo os critérios da DSM IV. Os dados sociodemográficos foram expressos em média (desvio padrão). Comparou-se os escores do MEEM e CAMCOG dos pacientes com psicose, demência e transtorno do humor utilizandose o teste t de Student e Anova.

Resultados. Da população total - idade: 70,87 (dp=6,6l); $m=32 ; f=54 ;$ analfabetos $=41$, escolarizados $=45-17(19,7 \%)$ tinham demência, II (I2,8\%) psicose e 58 (67,4\%) transtorno do humor. O MEEM do grupo total foi de 21,07 (dp=6,6 I) e CAMCOG de 61,50 (dp=19,78). MEEM/CAMCOG por diagnósticos: Demência: 16,76 $(d p=6,25) / 48(d p=20,49)$; Psicose: 20,9 (dp=5,87) / 60,09 (dp=13,54); Transtornos do humor: 22,36 ( $\mathrm{dp}=5,49)$ / 66,03 ( $\mathrm{dp}=18,88)$. A pontuação do MEEM e CAMCOG de demenciados foi significativamente menor que a dos pacientes com transtorno do humor (Anova $p<0,01$ ). A pontuação de pacientes com psicose não se diferenciou da dos pacientes com demência e da dos pacientes com transtornos do humor $(p>0,05)$.

Conclusãä. A avaliação cognitiva breve permite uma diferenciação sindrômica entre depressão e demência, mas não entre depressão e psicose, em pacientes idosos de um ambulatório psiquiátrico que apresentam queixas de memória. Estes dados podem servir para a organização de um protocolo simples e de baixo custo para o atendimento da população em serviç̧os de saúde pública.

Unitermos: Neuropsicologia. Idoso. Cogniçã̃o. Depressão. Demência. Psicose.

\section{INTRODUÇÃO}

O crescimento populacional na faixa de idosos determina forte demanda sobre os serviços primários de saúde tanto em países desenvolvidos como nos países em desenvolvimento ${ }^{1-5}$. No Brasil, até $40 \%$ dos idosos procuram serviços de saúde ao longo de três meses ${ }^{6}$.

Demência e depressão são os transtornos neuropsiquiátricos mais comuns em idosos. A demência afeta cerca de $5 \%$ dos idosos aos 65 anos de idade e cerca de $20 \%$ daqueles com 80 anos ou mais ${ }^{7.8}$. Recente estudo mostrou que, no Brasil, a incidência de demência em idosos residentes na comunidade alcança a taxa de 13,8 por 1000 habitantes/ ano, sendo que para Doença de Alzheimer (DA) o índice é de $7,7^{9}$. Também a depressão tem taxas de prevalência entre $5 \%$ e $35 \%$, variando de acordo com o nível de gravidade ${ }^{10}$. A prevalência de sintomas depressivos na população acima de 65 anos de idade vivendo na comunidade varia entre $10,3 \%$ e 13,5\%" e no Brasil chega a $14,3 \%{ }^{12}$.

O diagnóstico diferencial entre demência e depressão é freqüentemente difíil e nem sempre excludente, o que evidencia a necessidade da utilização de instrumentos que permitam, por meio da avaliação do estado cognitivo, distinguir melhor ambas as condições.
Em geral os psiquiatras tendem a reconhecer mais a depressão e os neurologistas mais os problemas cognitivos na população idosa. Entretanto, muitas vezes essas condições estão associadas e são freqüentes os sintomas depressivos como forma de apresentação de um transtorno cognitivo, como na $\mathrm{DA}^{13-14}$. Inversamente, são também freqüentes as alterações cognitivas secundárias à alteração do humor, em quadros depressivos de início tardio e em transtorno depressivo maior, recorrente em idosos ${ }^{15}$.

A realização da avaliação cognitiva pode ser um instrumento útil na avaliação global do paciente idoso, permitindo ao clínico geral, psiquiatra, neurologista ou geriatra obter informações que subsidiem tanto o diagnóstico etiológico do quadro em questão quanto o planejamento e execução das medidas terapêuticas e de reabilitação a serem realizadas em cada caso. Infelizmente, esta não é uma prática de rotina no Brasil em serviços de saúde primária e mesmo secundária, seja em Psiquiatria, Neurologia ou Geriatria.

Assim, a avaliação cognitiva de uma amostra de pacientes de um ambulatório de idosos com transtornos psiquiátricos pode trazer informações quanto à diferenciação clínica nestes casos. Este estudo preliminar teve por objetivo comparar o desempenho cognitivo de 
REYS BN ET AL.

idosos com psicose, depressão e demência. Tivemos por hipótese que, mesmo com queixas de memória, os pacientes com depressão e com psicose que não apresentassem demência do ponto de vista clínico teriam um desempenho cognitivo melhor do que os indivíduos com demência.

\section{Métodos}

\section{Participantes}

Todos os pacientes com idade acima de 60 anos avaliados consecutivamente durante os anos de $200 \mathrm{I}$ a 2004 em um ambulatório de Psiquiatria Geriátrica do Rio de Janeiro e que se queixavam de problemas de memória durante a consulta com um psiquiatra ou que geravam a suspeita no médico sobre este problema foram encaminhados para testagem cognitiva. Do total de 92 pacientes inicialmente vistos, 6 foram excluídos da amostra por epilepsia $(n=3)$, retardo mental $(n=2)$ e transtorno de personalidade $(n=1)$, permanecendo 86 indivíduos, sendo 32 masculinos e 54 femininos.

\section{Local do estudo}

O CPRJ é uma unidade pública de saúde mental pertencente à estrutura da Secretaria Estadual de Saúde do Rio de Janeiro. A unidade está situada no centro da cidade e funciona como um dos quatro grandes pólos psiquiátricos da capital do Rio de Janeiro, sendo uma referência para $\mathrm{O}$ atendimento de emergência e internação.

A área programática do ambulatório do CPRJ cobre o atendimento de aproximadamente $5 \%$ da população total de idosos da cidade do Rio de Janeiro, ou seja, 37.255 pessoas acima de 60 anos, sendo 26.945 (72,32\%) acima de 65 anos $^{16}$. Até dezembro de 2004, 89I pacientes idosos estavam cadastrados em atendimento nesta unidade. Portanto, a amostra avaliada neste estudo abrangia 9,6\% do total de idosos em atendimento.

\section{Instrumentos}

Criou-se no CPRJ uma rotina para exame de pacientes idosos descrita adiante. A partir desses procedimentos de rotina, foi realizada uma avaliação retrospectiva dos prontuários.

Os pacientes foram submetidos a uma anamnese semi-estruturada psiquiátrica e, em seguida, à avaliação cognitiva utilizando-se 0 miniexame do estado mental (MEEM) ${ }^{17}$ e o Cambridge Cognitive Examination 18. O MEEM avalia a orientação, atenção, concentração, memória, cálculo, linguagem e práxis. $\mathrm{O}$ escore varia de 0 a 30.0 ponto de corte abaixo do qual no MEEM considera-se a possibilidade de demência é 24 para os pacientes escolarizados e 18 para os analfabetos $^{8}$. O CAMCOG é a seção B do CAMDEX (Cambridge Examination for Mental Disorders of the Elderly $)^{18}$, uma entrevista estruturada para o diagnóstico de doenças neuropsiquiátricas relativas ao envelhecimento, principalmente o diagnóstico de demência em estágios iniciais. É um teste neuropsicológico que inclui, além dos 19 itens do MEEM, questões que se referem à percepção e ao pensamento abstrato. Neste teste são avaliadas as seguintes áreas da cognição: orientação, linguagem, memória, atenção, cálculo, praxias, pensamento abstrato e percepção. O CAMCOG consiste em 60 itens com escore máximo de 107 pontos, sendo o ponto de corte sugerido pelos autores

\section{Tabela I - Média (desvio padrão) dos resultados do MEEM e CAMCOG por diagnóstico e idade}

\begin{tabular}{|c|c|c|c|c|}
\hline & $\begin{array}{l}\begin{array}{c}\text { Demência } \\
\mathrm{N}=17\end{array} \\
\end{array}$ & $\begin{array}{c}\text { Transt. do humor } \\
N=58\end{array}$ & $\begin{array}{l}\text { Psicose } \\
\mathrm{N}=\mathrm{II}\end{array}$ & $\begin{array}{l}\text { Anova } \\
\text { p valor }\end{array}$ \\
\hline Idade & $75,94(6,87)$ & $70,17(6,10)$ & $67,63(4,86)$ & 0,337 \\
\hline MEEM & $16,76(6,25)$ & $22,36(5,49)$ & $20,90(5,87)$ & 0,002 \\
\hline CAMCOG & $48(20,49)$ & $66,03(18,88)^{*}$ & $60,09(13,54)$ & 0,003 \\
\hline
\end{tabular}

MEEM: miniexame do estado mental

CAMCOG: Cambridge Cognitive Examination

* Somente 54 pacientes com transtorno do humor realizaram o CAMCOG

79/80 para discriminar entre pacientes com demência e sujeitos normais. Foi utilizada a versão brasileira validada do CAMCOG ${ }^{19}$. Os profissionais que aplicaram os testes não conheciam o diagnóstico dos pacientes.

Os diagnósticos seguiram os critérios da DSM IV20 sem a utilização dos resultados dos testes aplicados e foram realizados exames complementares bioquímicos e de imagem (tomografia computadorizada ou ressonância magnética) sempre que necessários para a elucidação diagnóstica. Apenas 19 dos 86 pacientes da amostra têm resultados de neuroimagem. Por conta disso, os pacientes deste estudo foram agrupados em três categorias de acordo com o diagnóstico sindrômico: psicose, demência e transtorno do humor.

\section{Análise estatística:}

Os dados sociodemográficos foram expressos em média (desvio padrão).

A pontuação do MEEM e do CAMCOG dos pacientes nos três grupos sindrômicos foi comparada utilizando-se o teste t de Student e Anova (Tuckey post hoc).

O estudo foi aprovado pelo Comitê de Ética em Pesquisa do CPRJ e todos os pacientes assinaram o consentimento livre e esclarecido.

\section{Resultados}

A amostra total tinha média de idade de 70,87 $(\mathrm{dp}=6,61)$ anos e $4 \mathrm{I}$ eram analfabetos e 45 escolarizados. Demência foi diagnosticada em I7 pacientes (I9,7\%), psicose em II (I2,8\%) e transtorno do humor em 58 (67,4\%). A pontuação do MEEM do grupo total foi de 21,07 $(6,61)$ e a do CAMCOG foi $61,50(\mathrm{dp}=19,78)$.

A Tabela I mostra a distribuição dos resultados do MEEM e do CAMCOG e a média de idade dos três grupos por diagnóstico. A idade não apresentou diferença estatisticamente significativa entre os grupos estudados. A pontuação do MEEM e do CAMCOG de pacientes com demência é significativamente menor que a pontuação observada nos pacientes com transtorno do humor (Anova $p<0,01$ ). A pontuação nas escalas cognitivas dos pacientes com psicose não se diferencia da dos pacientes com demência e da dos pacientes com transtorno do humor $(p>0,05)$. Dos 58 pacientes com transtorno do humor, quatro não conseguiram concluir a testagem no CAMCOG, todos por terem alegado "nervosismo" ou incapacidade para fornecer as respostas.

O MEEM apresentou uma diferença significativa entre o grupo com demência e o grupo com transtorno do humor (Tuckey $q=5,042$, 
$p<0,01$ ), mas não entre estes e o grupo com psicose (demência versus psicose: Tuckey $q=2,661, p>0,05$; transtorno do humor versus psicose: Tuckey $q=1,098, p>0,05)$. Os mesmos achados foram observados no CAMCOG (demência versus transtorno do humor: $q=4,9 \mid 9, p<0,01$; demência versus psicose: $q=2,370, p>0,05$; transtorno do humor versus psicose: $q=1,363 ; p>0,05$ ).

\section{Discussão}

As doenças que causam dificuldades de memória em idosos, freqüentemente negligenciadas pelas equipes nos estabelecimentos de saúde, têm diagnóstico diferencial firmado pela avaliação neuropsicológica, favorecendo a detecção precoce, acompanhamento e tratamento dos transtornos cognitivos.

Este estudo apresenta dados preliminares sobre uma amostra de idosos com queixa de memória que procuraram um ambulatório de Psiquiatria, sem que o problema cognitivo fosse a primeira queixa na maior parte dos casos. Nossos resultados mostram uma diferença estatisticamente significativa da avaliação neuropsicológica breve de idosos com demência leve a moderada e de idosos com depressão, mas não entre idosos com depressão e com psicose. Isso pode ser um indicador de que uma simples e rápida testagem cognitiva já permite separar o comprometimento cognitivo dos pacientes com demência de outras categorias comuns em atendimentos psiquiátricos e neurológicos. Pode assim contribuir significativamente para a maior precisão do diagnóstico e para o planejamento terapêutico e das atividades de reabilitação, principalmente em um contexto de países em desenvolvimento nos quais procedimentos mais caros (neuroimagem) são difíceis de serem obtidos.

As queixas atuais de memória de idosos têm validade preditiva para demência e podem evidenciar comprometimento cognitivo passado e futuro ${ }^{21,14}$. As queixas de memória em idosos na comunidade aumentam em quatro vezes o risco de desenvolvimento de demência quando comparados a indivíduos sem queixa de memória ${ }^{22}$. No contexto brasileiro de um ambulatório de Psiquiatria Geriátrica, 72,4\% dos indivíduos que se queixaram de problemas de memória tinham efetivamente um diagnóstico de demência ${ }^{23}$.

Encontramos em nosso estudo um grande número de pacientes com queixas de memória e depressão. Dos 86 pacientes testados, 58 $(67,4 \%)$ apresentavam transtorno do humor. Este dado pode ser uma característica decorrente do fato do ambulatório do CPRJ ser exclusivamente psiquiátrico, sendo de se esperar que os pacientes com problemas primários de memória se dirijam preferencialmente a ambulatórios de Neurologia. Nestes, a prevalência de depressão como causa de síndrome demencial é significativamente menos freqüente $(11,5 \%)^{24,25}$. Dos pacientes com depressão em nosso estudo, 34,5\% ( $n=20)$ tinham pontuação no MEEM e no CAMCOG dentro da média observada no grupo dos pacientes com demência, sem, no entanto, apresentarem problemas clínicos e de atividades de vida diária que confirmassem um diagnóstico sindrômico de demência. Somente uma avaliação mais aprofundada e também longitudinal destes indivíduos pode precisar o diagnóstico, uma vez que a demência pode ser clinicamente evidente a partir de um tempo maior de observação ${ }^{14}$.
A presença de depressão diminui a validade preditiva das queixas de memória quanto ao diagnóstico de demência ${ }^{21}$. Idosos com prejuízo cognitivo podem melhorar seu desempenho em seguida ao tratamento com antidepressivos, particularmente na memória e funções executivas sem, contudo, alcançar níveis normais em domínios específicos. Este é o subgrupo de pacientes com depressão tardia mais propenso ao desenvolvimento de demência ${ }^{26-27}$.

O presente estudo apresenta uma série de pontos passíveis de crítica e que merecem consideração. Não utilizamos em nosso estudo escalas de avaliação para determinar a intensidade de sintomas depressivos e psicóticos nos três grupos e tampouco avaliamos a influência que o tempo de doença pudesse ter tido nos resultados. O número menor de indivíduos no grupo com psicose também pode ter tido influência nos resultados negativos para diferenciá-los dos demais grupos. A utilização das escalas poderá gerar dados importantes quanto ao grau de comprometimento psicopatológico e o grau de comprometimento cognitivo observado. No entanto, o diagnóstico clínico seguindo os critérios da DSM IV para as síndromes aproxima os nossos resultados do que efetivamente acontece na vida real dos clínicos envolvidos em atendimento primário e secundário no Brasil.

Em nosso meio, a questão da baixa escolaridade da população atendida nos serviços de saúde torna-se um importante fator a ser observado. Na amostra utilizada neste trabalho, encontramos 41 indivíduos analfabetos (47,6\%). Tal fato mostra-se relevante tanto no que diz respeito à aplicação da testagem cognitiva, quanto em relação à interpretação dos resultados das mesmas.

Outrossim, o diagnóstico sindrômico não contou com a neuroimagem como exame complementar. De qualquer modo, o desempenho cognitivo deste grupo foi significativamente superior ao observado no grupo com demência.

\section{Conclusão}

A avaliação cognitiva breve permite uma diferenciação sindrômica entre depressão e demência, mas não entre depressão e psicose, em pacientes idosos de um ambulatório psiquiátrico que apresentam queixas de memória. Estes dados podem servir para a organização de um protocolo simples, racional e de baixo custo para o atendimento da população em serviços de saúde pública.

Conflito de interesse: não há.

\section{SUMMARY}

Brief cognitive evaluation and diagnosis of dementia, DEPRESSION, AND PSYCHOSIS IN THE ELDERLY

OBJECTNE. To evaluate the cognitive performance of the elderly with psychosis, depression and dementia in a mental health outpatient unit.

Methods. The Mini-Mental State Examination (MMSE) and the Cambridge Cognitive Examination (CAMCOG) were applied to 86 patients above 60 years of age referred for evaluation due to memory complaints. Patients were diagnosed according to DSMIV criteria. Socio-demographic data were expressed as means (standard deviation); MMSE and CAMCOG score of patients with 
REYs BN ET AL.

psychosis, dementia and mood disorders were compared using Student's T test and ANOVA.

RESULTS. Of the total sample (age: $70.87(s d=6.61)$; male=32; female $=54$; illiterate $=41$, educated $=45 ;$ 17(19.7\%) had dementia, I I (12.8\%) psychosis and 58 (67.4\%) mood disorders. The MMSE of the total sample was 21.07 $(s d=6.61)$ and the CAMCOG $=61.50$ $(s d=19.78)$. MMSE/CAMCOG of each diagnosis: Dementia: 16.76 $(s d=6.25) / 48(s d=20.49) ; \quad$ Psychosis: $20.9 \quad(s d=5.87) / 60.09$ $(s d=13.54)$; mood disorders: 22.36 ( $s d=5.49) / 66.03(s d=18.88)$. The MMSE and CAMCOG scores of patients with dementia were significantly lower than those of patients with mood disorders (ANOVAp $<0.01$ ). The score of patients with psychosis was not different from scores of those with dementia and mood disorders $(p>0.05)$.

CONCLUSION. A brief cognitive evaluation permits a differentiation between dementia and depression but not between psychosis and depression in elderly patients of a psychiatric outpatient unit, who had complained ofmemory impairment. These data may be useful to develop an easy and low cost protocol to attend the population of public health services. [Rev Assoc Med Bras 2006; 52(6): 40 I-4]

KeY wORDS: Neuropsychology. Elderly. Cognition. Depression. Dementia. Psychosis.

\section{REFERÊNCIAS}

I. Monnot M, Brosey M, Ross E. Screening for dementia: family caregiver questionnaires reliably predict dementia. J Am Board Fam Pract. 2005; | 8:240-56.

2. PressleyJC, Trott C, Tang M, Durkin M, Stern Y. Dementia in communitydwelling elderly patients: a comparison of survey data, medicare claims, cognitive screening, reported symptoms, and activity limitations. J Clin Epidemiol. 2003;56:896-905.

3. Lin RT, Lai CL, Tai CT, Liu CK, Yen YY, Howng SL. Prevalence and subtypes of dementia in southern Taiwan: Impact of age, sex, education, and urbanization. J Neurol Sci. 1998; 160:67-5.

4. Ramirez EL, Pallas AJM, Domenech VJ, Pueyo AT, Pena CC. Bargallo EV. Investigadores del proyecto Cuida'l (Detection Of Cognitive Impairment in the Population of Persons Older than 64 Years: First Phase of the Cuida'l Project). Aten Primaria. 2003;32:6-14.

5. Vas CJ, Pinto C, Panikker D, Noronha S, Deshpande N, Kulkarni L, et al. Prevalence of dementia in a urban Indian population. Int Psychogeriatr. 200 I; 13:439-50

6. Laks J, Marinho V. O ambulatório de psiquiatria geriátrica do Instituto de Psiquiatria da UFRJ: objetivos, casuística e características do atendimento. J Bras Psiquiatr. 1997;46:543-6.

7. Jorm AF, Jolley D. The incidence of dementia: a meta-analysis. Neurology. 1998;51:728-33.

8. Herrera E, Caramelli P, Silveira ASB, Nitirni R. Epidemiologic survey of dementia in a community-dwelling Brazilian population. Alzheimer Dis Assoc Disord. 2002; 16:103-8.

9. Nitrini R, Caramelli P, Herrera Jr E, Bahia VS, Caixeta LF, Radanovic M, et al. Incidence of dementia in a community-dwelling brazilian population. Alzheimer Dis Assoc Disord. 2004; 1 8:24I-6.
10. Almeida OP, Garrido R, Tamai S. Unidade para idosos (UNID) do Departamento de Saúde Mental da Santa Casa de São Paulo: características clínicas de pacientes atendidos em nível ambulatorial. J Bras Psiquiatr. 1998;47:291-6.

I I. Snowdon J. How high is the prevalence of depression in old age. Rev Bras Psiquiatr Supl. 2002;24:S42-S47.

12. Blay SL, Bickel H, Cooper B. Mental illness in cross- national perspective. Results from a Brazilian and German community survey among the elderly. Soc Psychiatr Epidemiol. 1999; 26:245-5I.

13. Lyketsos CG, Steele C, Baker L, Galik E, Kopunek S, Steinberg M, et al. Major and minor depression in Alzheimer's disease: prevalence and impact. J Neuropsychiatry Clin Neurosci. 1997;9:556-6I.

14. Edwards ER, Lindquist K, Yaffe K. Clinical profile and course of cognitively normal patients evaluated in memory disorders clinics. Neurology 2004;62: 1639-42.

15. Rapp MA, Dahlman K, Sano M, Grossman HT, Haroutunian V, Gorman JM. Neuropsychological differences between late-onset and recurrent geriatric major depression. Am J Psychiatry. 2005; | 62:69|-8.

16. Armazém de Dados. Tabela 4.2.6: População residente, por grupos de idade e sexo, segundo áreas de planejamento, regiões administrativas e bairros. 2000 [online]. Disponível em: http:/ www.armazemdedados. rio.ri.gov.br/index.htm.

17. Folstein MF, Folstein SE, McHugh PR. "Mini-Mental State": a practical method for grading the cognitive state of patients for the clinician. J Psychiatr Res. 1975; I 2: 189-98.

18. Roth M, Huppert FA, Tym E, Mountjoy CQ. CAMDEX: The Cambridge examination for mental disorder of the elderly. Cambridge: Cambridge University Press; 1986.

19. Bottino CMC, Almeida OP, Tamai S, Forlenza OV, Scalco MZ, Carvalho IAM. Entrevista estruturada para o diagnóstico de transtornos mentais em idosos - CAMDEX - The Cambridge examination for mental disorders of the elderly. Brazilian version (translated and adapted on behalf of the editors). Cambridge: Cambridge University Press; 1999.

20. American Psychiatric Association. Diagnostic and statistical manual of mental disorders(DSM-IV). $4^{\text {th }}$ ed. Washington: APA; 1994.

21. Jorm AF, Christensen H, Korten AE, Jacomb PA, Henderson AS. Memory complaints as a precursor of memory impairment in older people: a longitudinal analysis over 7-8 years. Psychol Med. 200 I;3 I:44 I-9.

22. Tobiansky R, Blizard R, Livingston G, Mann A. The Gospel Oak Study stage IV: the clinical relevance of subjective memory impairment in older people. Psychol Med. 1995;25:779-86.

23. Almeida OP. Queixa de problemas com a memória e o diagnóstico de demência. Arq Neuropsiquiatr. 1998;56:4 I2-8.

24. Vale FAC, Miranda SJC. Clinical and demographic features of patients with dementia attended in a tertiary outpatient clinic. Arq Neuropsiquiatr. 2002:60:548-52.

25. Silva DW, Damasceno BP. Demência na população de pacientes do Hospital das Clínicas da Unicamp. Arq Neuropsiquiatr 2002;60:996-9.

26. Alexopoulos GS, Myers BS, Young RC, Kalayam B, Kakuma T, Gabrielle $M$, et al. Executive dysfunction and long-term outcomes of geriatric depression. Arch Gen Psychiatry. 2000;57:285-90

27. Butters MA, Becker JT, Nebes RD, Zmuda MD, Mulsant BH, Pollock BG, et al. Changes in cognitive functioning following treatment of late-life depression. Am J Psychiatry. 2000; 1 57: 1949-54.

Artigo recebido: 07/10/05

Aceito para publicação: 02/02/06 